\title{
Integrating problem solving and knowledge construction through dual mapping
}

\author{
Bian $\mathrm{Wu}^{*}$ \\ Faculty of Education \\ The University of Hong Kong, Hong Kong \\ E-mail: covenwu@hku.hk
}

\section{Minhong Wang}

Faculty of Education

The University of Hong Kong, Hong Kong

E-mail: magwang@hku.hk

*Corresponding author

\begin{abstract}
Learning through problem solving has been regarded as an important approach to constructivist learning. However, how practice and knowledge reciprocate each other has not been sufficiently examined and remains implicit. Although problem-based learning is increasingly used in medical education and other domains, there is a concern about its weakness in general study design in relation to its impact on learners' knowledge base. Considering the complex cognitive processes involved in learning through problem solving, this study proposes a dual mapping learning environment, serving as a visual affordance for improving problem solving and underlying knowledge construction processes as well as the transformation between the two.
\end{abstract}

Keywords: Problem solving; Knowledge construction; Visualization; Dual mapping; Cognitive tool

Biographical notes: Bian Wu is a doctoral candidate of Faculty of Education, The University of Hong Kong. His research interests include problem-based learning, clinical problem solving, and technology-enhanced learning. He has completed all of the requirements for the doctoral degree with the exception of the dissertation.

Dr. Maggie Minhong Wang is an Associate Professor in the Faculty of Education, The University of Hong Kong. She has been involved in multiple disciplinary research in the areas of technology-enhanced learning, complex problem solving and learning, knowledge management, adult learning and human performance, , and artificial intelligence. She has published papers in Computers \& Education, Information \& Management, IEEE Transactions on Education, Educational Technology \& Society, Innovations in Education \& Teaching International, Expert Systems with Applications, Knowledge-based Systems, Journal of Knowledge Management, among others. She is the Editorin-Chief of Knowledge Management and E-Learning: an International Journal. She also serves on the editorial board of several international journals including Educational Technology Research and Development, and Educational Technology \& Society. More details can be found at http://web3.edu.hku.hk/magwang/. 


\section{Introduction}

Learning through problem solving has received increased attention in support of constructivist learning, especially in ill-structured domains such as medical education and engineering design (Jonassen, 1999). Through problem solving practice, learners continually learn and update their knowledge based on their experiences with novel problems and situational demands (Brandsford, Pellegrino, \& Donovan, 1999). For effective learning through problem solving, practical experience and knowledge construction should highly integrate and reciprocate each other (DeGrave, Boshuizen, \& Schmidt, 1996; Kinchin, Cabot, \& Hay, 2008). However this is difficult to achieve. Both problem solving experience and knowledge construction are complex cognitive processes, which cannot be easily captured and mastered. Computer-based cognitive tools can help to externalize and elicit the complex structure and process of human cognition using visual representations, and engage learners in representing, manipulating and reflecting on what they know (Wang \& Jacobson, 2011; Wang, Peng, Cheng, Zhou, \& Liu, 2011). However, there is a lack of studies on such cognitive support to facilitate learning through problem solving (Wu \& Wang, 2010).

To deal with the challenge, this study has designed and implemented a dual mapping learning environment, to help learners to visualize their problem solving and knowledge construction processes, and more importantly, to support the transformation between the two. Medical education is selected as the domain of this study, where problem-based learning is regarded as crucial to learning and expertise development in this field. Although problem-based learning is increasingly used in medical education, there is a concern about its weakness in general study design in relation to its impact on learners' knowledge base (Dochy, Segers, Van den Bossche, \& Gijbels, 2003). This study aims to address the challenge by investigating how learning through problem solving can be supported by the design of a technology-enhanced learning environment that makes complex cognitive processes visible for problem solving and knowledge construction. The design is focused on a dual mapping learning environment that involves concept mapping and argument mapping tools to represent learners' problem solving process and the underlying domain knowledge in visual formats, in addition to other functions to support the problem solving and learning process. To evaluate the proposed design, the dual mapping learning environment has been implemented, used and evaluated by learners from two medical schools in China.

\section{Conceptual framework}

The design of the proposed learning environment refers to cognitive theories and models proposed by Mayer (1996) and Dougherty, Thomas, and Lange (2010), as shown in Fig. 1. Mayer's (1996) SOI (Selecting, Organizing, and Integrating) model describes three sequential cognitive processes, i.e., selecting relevant information for further processing in working memory (WM); organizing incoming information into a coherent representation in WM; and integrating the new information with the learner's prior knowledge in long-term memory (LTM). This model depicts the process and mechanism of knowledge construction. Considering the limited capacity of human information processing, mental models are needed to externalize mental activities and to retain knowledge in LTM. In this study, concept mapping is used as a typical mental model to 
represent the domain knowledge structure for internalization into LTM. The domain knowledge structure is subject to update and reorganization based on new understanding.

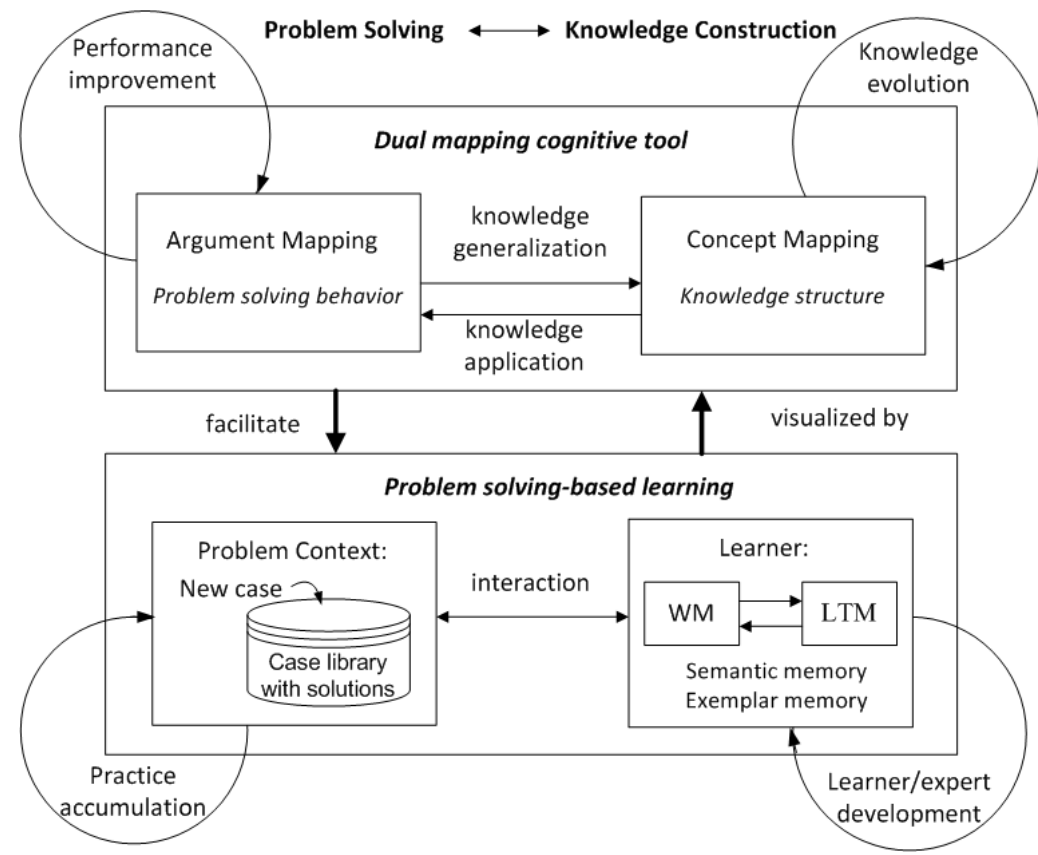

Fig. 1. Conceptual framework of the dual mapping learning environment

Dougherty, Thomas, and Lange (2010) presented a detailed computational model, to outline the relationships among ecological environment (i.e., perceptual information), memory system (categorized as working memory, exemplar memory, and semantic memory), and problem solving behavior (including hypothesis generation, probability judgment, and hypothesis testing). Their model depicts the process and mechanism of hypothesis-led problem solving. Based on this model, the problem solving process starts from observed data triggering traces from exemplar memory; then probes from semantic memory might generate hypotheses with constraints; finally, judgments may occur along with justification of the hypotheses. The process is iterative and dynamic, where hypotheses can be updated when new data are encountered. In this study, argument mapping is used to represent the problem solving process including hypothesis generation, justification, and diagnostic conclusion.

For representation of cognitive processes in problem solving and knowledge construction, two types of cognitive tools - argument mapping and concept mapping, are found to be directly relevant to this study. Cognitive tools are usually computer-based tools for engaging and facilitating cognitive processing. Argument mapping is a visual representation of the structure of an argument in informal logic. It can be used to explore reasoning and decision making process in problem solving practice (Fox et al., 2007). Concept mapping is a visual representation of concepts and their relationships. It is mainly for representing and organizing domain knowledge, providing a system view of knowledge structure (Novak \& Gowin, 1984). In most existing studies, concept mapping and argument mapping have been used separately for different learning contexts. This study has integrated them with further exploration to support the problem solving and learning process. 


\section{Design of the dual mapping learning environment}

Based on the conceptual framework, a Web-based dual mapping learning environment is designed and implemented to support learning through problem solving. As outlined in Fig. 2, the system consists of three major components: an exploratory problem context, a dual mapping cognitive tool as a visual affordance, and pedagogical scaffolds for adaptive learning support. Learners can navigate between the two windows of the problem context and the dual mapping cognitive tool iteratively. When facing difficulties, they can request support by clicking the help buttons to view relevant help dialog boxes.

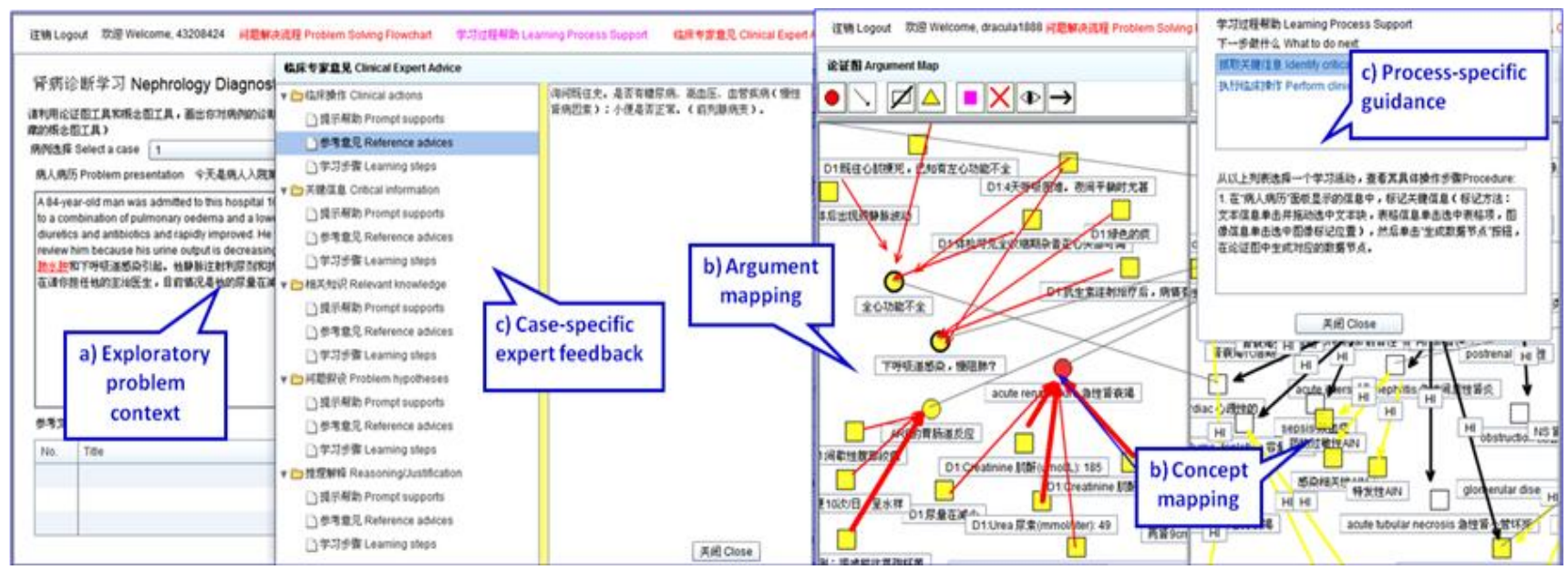

Fig. 2. Interfaces of the dual mapping learning system

The learning process may start from access to the problem case by the learner who is usually equipped with semantic and exemplar memory relevant to the problem domain. Based on the problem information, the learner may start the problem solving process and represent the key activities such as hypothesis generation, hypothesis justification, and diagnostic conclusion in an argument map. In solving the problem, the learner may need to trigger relevant domain knowledge stored in their LTM, which can be externalized into a concept map for easy recall and retrieval. The concept map is subject to update and extension by the learner throughout the problem solving and learning process.

\subsection{Exploratory problem context}

For each clinical case, the patient information including texts, charts, and images is organized into different categories and subcategories. Learners may access initial information and perform clinical actions such as ordering a specific lab test to achieve additional information to explore and solve the problem. Most clinical cases are progressive and patient information is achieved in a time sequence, instead of one snapshot.

\subsection{Dual mapping cognitive tool}

The dual mapping cognitive tool is central to the learning environment. It involves concept mapping and argument mapping for visualizing and facilitating the problem solving and knowledge construction processes. Argument mapping helps learners to 
sharpen their problem solving skills through building coherent and well-grounded argumentation structure. Concept mapping pulls out medical science and clinical knowledge, and helps learners to conceptualize contextual knowledge. It provides anchored points for problem solving based on relevant domain knowledge, and for construction of new understanding based on prior knowledge.

Considering the complexity caused by a number of interactive activities involved in the problem solving and knowledge construction activities, the whole process is decomposed into the following five steps.

Critical information capture. After the learner accesses the clinical case and identifies its critical information, he/she may start the problem solving process by generating relevant data nodes in the argument map, i.e., by dragging the data or information from the problem context window to the argument map.

Knowledge recall and update. To analyze and solve the problem, the learner needs to recall the domain knowledge which can be represented in a concept map. He/She may update the concept map when needed.

Hypotheses generation. Based on the case information and relevant domain knowledge, the learner can make hypotheses, and add them as hypothesis nodes into the argument map. Where appropriate, the hypothesis nodes can be generated based on relevant concepts from the concept map.

Reasoning and justification. The learner can evaluate a hypothesis via reasoning and justifications. The action can be represented as reasoning links between hypothesis nodes and relevant data nodes in the argument map. The learner may justify or explain the reasoning action by adding brief text to the reasoning links. In addition, the learner can generate one or more evidence nodes, and link them to information of external references to support the reasoning.

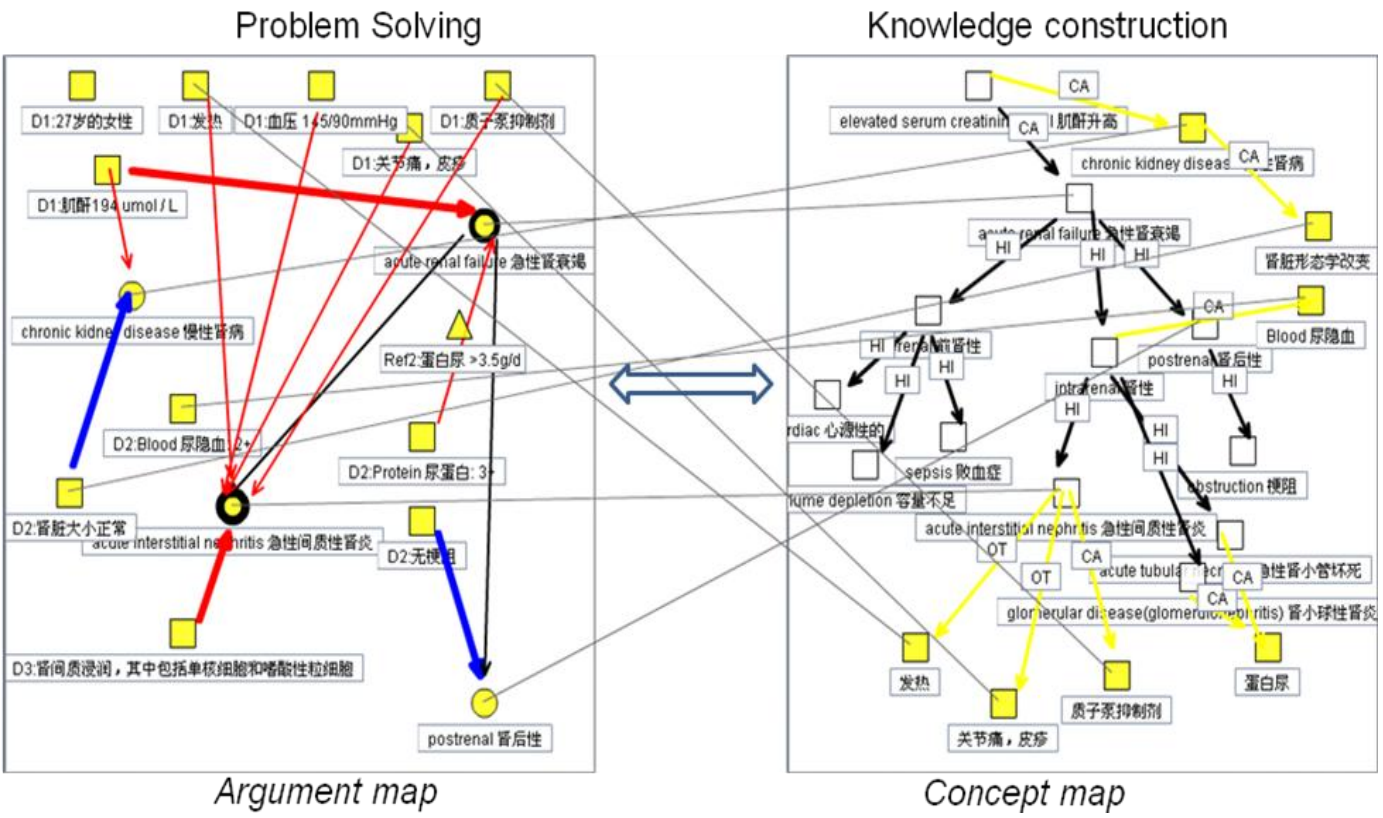

Fig. 3. Argument mapping and concept mapping with their interactions 
Diagnostic conclusion. Based on the generated and justified hypotheses, the learner can make a diagnostic conclusion, represented as a diagnostic node in the argument map. In the meantime, the learner can reflect on the cognitive activities during or after the problem solving process. New knowledge learnt from problem solving with the case can be integrated into prior knowledge by updating or reorganizing the concept map to expand or improve existing understanding.

The details of the argument mapping and concept mapping are presented in Fig. 3. To highlight the interactions between problem solving and knowledge construction, hypothesis nodes, reasoning links, and evidence nodes in the argument map can be linked with relevant concept nodes in the concept map to indicate the knowledge underlying the hypotheses and reasoning actions. Other features of the dual mapping cognitive tool include zooming in/out the maps; showing/hiding explanations or evidence nodes; and folding/unfolding sub-nodes of a selected node in a concept map.

\subsection{Scaffolding support}

To facilitate learners throughout the complex process in problem-solving learning, scaffolding support is provided. First, fundamental knowledge of the problem domain is represented in a concept map, provided as an anchoring point for learners to construct their knowledge. Learners can refine or reorganize the map based on their existing understanding and new knowledge learnt from the problem solving practice. Second, process-specific learning guidance is provided to help learners to go through a number of activities in problem-solving learning. By a click on the process-specific help button, guidance or suggestions such as the next appropriate action can be recommended to learners according to their current progress. Third, case-specific feedback and hints based on expert knowledge are provided for solving the case. The feedback covers the main issues for solving the problem case including clinical actions, critical information, hypotheses, reasoning and justifications, and relevant knowledge concepts. Moreover, a review of the case provided by domain experts can be accessed by learners once they complete the problem solving process with the case.

\section{Evaluation of the dual mapping learning environment}

To evaluate the proposed dual mapping learning environment, a series of studies and analyses were arranged. At the initial stage of the project, the evaluation was focused on learners' perception and reaction towards the system as reported in this paper. The assumption is that unless the proposed learning environment is properly designed and implemented to the extent that learners find it useful and acceptable, further exploration on the effect of the dual mapping learning environment may not produce reliable and meaningful results.

Twenty-nine students $(65.5 \%$ female and $34.5 \%$ male) from the two aforesaid medical schools were recruited as volunteer participants for an online learning program for learning with four clinical cases in kidney disease using the dual mapping learning system. They were in the third year or higher in their seven-year medical school curriculum (five years of undergraduate study plus two years of graduate study) with fundamental medical knowledge but little clinical experience. Most of them reported to have intermediate $(41.4 \%)$ or good $(41.4 \%)$ computer skills, and have a neutral $(41.4 \%)$ or positive $(41.4 \%)$ intention to use online learning application. The participants completed the four-week online learning program in their free time, and participated in a 
pre-test, a post-test, a survey, and interviews for evaluation of the system. One instructor from the medical school was invited to participate in this study to provide necessary support to learners.

The learning output was assessed based on the tests and dual mapping records. The paired sample $t$ test indicated that there was no significant difference between the pre-test score and post-test score within the four-week learning period. On the other hand, the dual mapping records generated by the learners from the first and the final case were assessed by two domain experts. A significant improvement was found from the first case to the last case, significant at the .01 level. The effect size (Cohen, 1998) was .5, which indicated moderate progress in learning performance reflected in the mapping records. The inter-rater reliability of dual mapping assessment by the two raters was .91, significant at the .01 level. Moreover, the dual mapping scores of the last case were found to be significantly correlated with the post-test results $(r=.421, \mathrm{p}<.05)$.

The survey questionnaire was designed to examine students' response to perceived usefulness (USE) and ease of use (EOU) of the overall system (OVER) and its main functions including the exploratory problem context (EPC), the dual mapping tool (DMT), and the scaffolding support (SS). The items measuring the constructs were adopted from related literature (Arbaugh, 2000; Venkatesh \& Davis, 2000). Responses to the items were ranked on a 5-point Likert scale (1 represented "strongly disagree" and 5 represented "strongly agree"). As shown in Table 1, the learners' perception towards the ease of use of problem context and learning support was found to be positive, while the perception on ease of use of the overall system and the dual mapping tool was weak positive.

Table 1

Perceived usefulness and ease of use of the dual mapping learning environment

\begin{tabular}{rrrrr}
\hline & OVER_USE & EPC_USE & DMT_USE & SS_USE \\
\hline Mean & 3.62 & 3.68 & 3.57 & 3.55 \\
Std. Deviation & .81 & .72 & .84 & .64 \\
\hline & OVER_EOU & EPC_EOU & DMT_EOU & SS_EOU \\
\hline Mean & 3.05 & 3.40 & 3.07 & 3.21 \\
Std. Deviation & .99 & .67 & .92 & .83 \\
\hline
\end{tabular}

In addition to the above quantitative evaluation, students' responses to open questions regarding the advantages and disadvantages of the developed system and suggestions on improvement were collected from interviews. Based on their qualitative feedback, it is found that most students felt the dual mapping learning system impressive and attractive. As one mentioned, "The system provides a very innovative and unique learning environment, giving learners a clear and systemic picture of the main tasks and knowledge involved in clinical problem solving." They also felt the learning guidance and expert feedback provided by the system very helpful for their self-directed learning, especially for checking missing points in their understanding and thinking. Some students also appreciated the clinical experiences provided by the dual mapping learning system, which they felt very useful for their professional development, but difficult to achieve in traditional education programs. On the other hand, students gave their comment on the limitations of the dual mapping learning system, together with suggestions for 
improvement. Many students felt that the current version of the system, in particular the dual mapping interface, was not quite user-friendly, mainly due to the complex processes involved in clinical problem solving and knowledge construction. They suggested the dual mapping process be simplified, or the description on the use of the system be improved with more instructions. Some students mentioned that the learning content can be improved by adding more typical cases. Some others suggested more references for fundamental knowledge of the problem domain be provided to the dual mapping learning system for easy access. Finally, most students expressed their clear intention to use the dual mapping learning system in their future study if the system can be improved as they suggested.

In the meantime, the instructor and domain experts participated in this study were interviewed for their comments on the dual mapping learning system and its impact on teaching and profession development. They expressed their clear interest and positive comments towards the innovative and systemic design of the dual mapping learning environment and its ability to stimulate learners' motivation and sense of autonomy for learning. In addition, they commented that most medical schools in China do not allocate sufficient time and resources for teaching sophisticated skills of problem solving; under this situation, there is a high demand to incorporate such kind of self-directed problemcentered learning environment into conventional education programs.

\section{Conclusion}

Learning through problem solving has been regarded as an important approach to constructivist learning. However, how practice and knowledge reciprocate each other has not been sufficiently examined and remained implicit, especially in technology-enhanced learning environment. Many online learning applications have been developed without alignment of learning with practical performance (Wang, Vogel, \& Ran, 2011). Considering the complex cognitive processes involved in problem solving and knowledge construction, this study proposed a dual mapping learning environment, serving as a visual affordance for learners to represent the problem solving process and underlying domain knowledge, as well as the transformation between the two.

The study presents the design, implementation, and evaluation of the proposed dual mapping learning environment for learning through clinical problem solving by medical students. It is a great challenge to represent and facilitate complex dynamic cognitive processes in clinical problem solving and knowledge construction. This project made an attempt to address the challenge by examining how a computer-based learning environment can be designed and implemented to support complex cognitive processes in learning through problem solving, as well as the effectiveness of the design learning environment.

The dual mapping learning environment has been designed by incorporating an exploratory problem context for exploration with problems and solutions, a dual mapping cognitive tool that supports articulation and reflection of cognitive processes in problem solving and learning, and scaffolding and coaching support that facilitates the complex problem solving and learning process. In particular, the design has focused on the dual mapping cognitive tool that incorporates argument mapping and concept mapping, and takes into account the hypothesis-led mechanism for problem solving and the SOI (selecting, organization, and integrating) model for knowledge construction. Furthermore, the two types of mapping processes have been integrated: concept maps retain conceptual knowledge for recall and update by problem solving practice, and argument maps 
externalize cognitive elements in problem solving practice for assimilating new understanding into existing knowledge structure. In this way, the designed dual mapping learning environment helps to achieve the goal of learning through problem solving, i.e., help learners to explore problems and solutions, articulate and reflect on their problem solving activities, and construct their knowledge by assimilating new understanding from problem solving practice.

The evaluation results showed that the dual mapping learning environment was perceived to be useful, innovative, and attractive. Overall it was well received by the learners. The learning effectiveness of the dual mapping learning environment was evidenced by the moderate learning progress achieved by the learners. The effectiveness was also perceived by the learners in presenting a clear picture of complex cognitive processes and fostering knowledge construction into a systemic structure. Moreover, the proposed learning environment was perceived helpful for stimulating learners' motivation and supporting self-directed learning.

Based on the feedback and suggestions from the learners, improvement of the dual mapping learning environment has been carried out with support of domain experts. Further evaluation and investigations on the effectiveness of the developed learning environment will be performed and reported in future work. On the other hand, the developed dual mapping learning environment provides a platform for further studies on how problem solving and knowledge construction interact and reinforce each other in some patterns.

\section{Acknowledgements}

This research is supported by Seeding Fund for Basic Research (No. 201011159210 and No. 201111159044) from The University of Hong Kong. The authors would thank Professor Jonathan Michael Spector for his valuable guidance and support to this project.

\section{References}

Arbaugh, J. B. (2000). Virtual classroom characteristics and student satisfaction with internet-based MBA courses. Journal of Management Education, 24(1), 32-54.

Brandsford, J. D., Pellegrino, J. W., \& Donovan, S. (1999). How people learn bridging research and practice. Washington, DC: National Academy Press.

Cohen, J. (1988). Statistical power analysis for the behavioral sceince. Hillsdale, NJ: Lawrence Erlbaum.

DeGrave, W. S., Boshuizen, H. P. A., \& Schmidt, H. G. (1996). Problem based learning: Cognitive and metacognitive processes during problem analysis. Instructional Science, 24(5), 321-341.

Dochy, F., Segers, M., Van den Bossche, P., \& Gijbels, D. (2003). Effects of problembased learning: A meta-analysis. Learning and Instruction, 13, 533-568.

Dougherty, M., Thomas, R., \& Lange, N. (2010). Toward an integrative theory of hypothesis generation, probability judgment, and hypothesis testing. In H. R. Brian (Ed.), Psychology of Learning and Motivation (Vol. 52, pp. 299-342). Academic Press.

Fox, J., Glasspool, D., Grecu, D., Modgil, S., South, M., \& Patkar, V. (2007). Argumentation-based inference and decision making--A medical perspective. Intelligent Systems, 22(6), 34-41.

Jonassen, D. (1999). Designing constructivist learning environments. In C. M. Reigeluth 
(Ed.), Instructional-design Theories and Models: A new paradigm of instructional technology (Vol. 2, pp. 215-239). Mahwah, New Jersey: Lawrence Erlbaum Associates.

Kinchin, I. M., Cabot, L. B., \& Hay, D. B. (2008). Visualising expertise: Towards an authentic pedagogy for higher education. Teaching in Higher Education, 13(3), 315326.

Mayer, R. E. (1996). Learning strategies for making sense out of expository text: The SOI model for guiding three cognitive processes in knowledge construction. Educational Psychology Review, 8(4), 357-371.

Novak, J. D., \& Gowin, D. B. (1984). Learning how to learn. New York: Cambridge University Press.

Venkatesh, V., \& Davis, F. D. (2000). A theoretical extension of the technology acceptance model: Four longitudinal field studies. Management Science, 46(2), 186204.

Wang, M., \& Jacobson, M. J. (2011). Knowledge visualization for learning and knowledge management (Editorial). Educational Technology \& Society, 14(3), 1-3.

Wang, M., Peng, J., Cheng, B., Zhou, H., \& Liu, J. (2011). Knowledge visualization for self-regulated learning. Educational Technology \& Society, 14(3), 28-42.

Wang, M., Vogel, D., \& Ran, W. (2011). Creating a performance-oriented e-learning environment: A design science approach. Information \& Management, 48(7), 260269.

Wu, B., \& Wang, M. (2011). Dual mapping for support of problem solving and knowledge construction. Proceedings of the 11th IEEE International Conference on Advanced Learning Technologies, Athens, Georgia, USA. 